\title{
Hydrogenated Castor Oil
}

National Cancer Institute

\section{Source}

National Cancer Institute. Hydrogenated Castor Oil. NCI Thesaurus. Code C83764.

A wax-like hydrogenated derivative of castor oil. Hydrogenated castor oil has many industrial applications. 